\title{
Intermuscular Hydatid Cyst Isolated from the Arm
}

\author{
Talal G*, Saudi F, Ouazzani N, Fekhaoui MR, Boufetal M, Reda-Allah B \\ and Saleh Berrada M \\ Department of Orthopedic Surgery and Trauma, BN SINA Hospital, Morocco
}

*Corresponding author: Talal Grimi, Department of Orthopedic Surgery and Trauma, IBN SINA Hospital, Morocco, Tel: +21234568578; Email: grimitalal@gmail.com

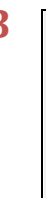

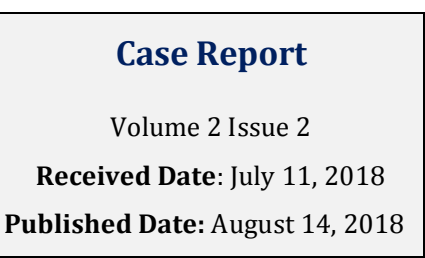

Case Report

Volume 2 Issue 2

Published Date: August 14, 2018

\section{Abstract}

Hydatid disease is an infestation caused by Echinococcus granulosus, which is still widespread in temperate zone countries. The muscoloskeletal localisation is extremely rare and represents a major challenge for surgeons because there are few cases reported in the literaturel. We report a case of a 30-year-old man with no medical antecedants, living in a rural area with a primary hydatid cyst located in the intermuscular space front of the arm.

Keywords: Echinococcus granulosus; Muscoloskeletal; Intermuscular Hydatid; Cyst Isolated

\section{Introduction}

Hydatid disease is an infestation caused by Echinococcus granulosus, which is still widespread in temperate zone countries. The muscoloskeletal localisation is extremely rare and represents a major challenge for surgeons because there are few cases reported in the literature. We report a case of hydatid cyst primary located in the intermuscular space front of the arm.

\section{Observation}

We report a 30-year-old man with no medical antecedants, living in a rural area, which had since 6 months pain and swelling of his right arm increasing volume gradually. The physical examination showed a mass about 15-8-6 cm from the right arm (Figure 1), there was no sign of an inflammatory process or lymphodenopathy. The range of motion of the elbow has been respected [1-3].
X-rays showed swelling of soft tissues, without calcification (Figure 2). Ultrasound revealed a cystic structure rounded, well defined in the intermuscular space of anterior right arm, which suggest an hydatid cyst. MRI revealed an hyposignal cystic mass on T1-weighted spin and sequences of hyperintensive T2 gradient overweight echo and the saturated fat of images, with presence of small hyposignal cysts on the surrounding parts of the mass (Figure 3). The Haemagglutination tests were positive for E. granulosus at a titre of $1 / 640$. The results of the chest $x$-ray, abdominal ultrasound, from the formula count blood pressure, sedimentation rate and Blood biochemistry was normal.

Due to the strong suspicion of the hydatid disease, complete surgical excision with a resection in entier bloc, without destroying the cyst wall was realized, the mass was strongly adherent to the surrounding muscle, the diagnosis has been well established, after histopathological examination which confirmed 


\section{Journal of Orthopedics \& Bone Disorders}

thediagnosis. A complementary anthelmintic treatment was given for six weeks and the patient is free from the disease during two years of follow-up.
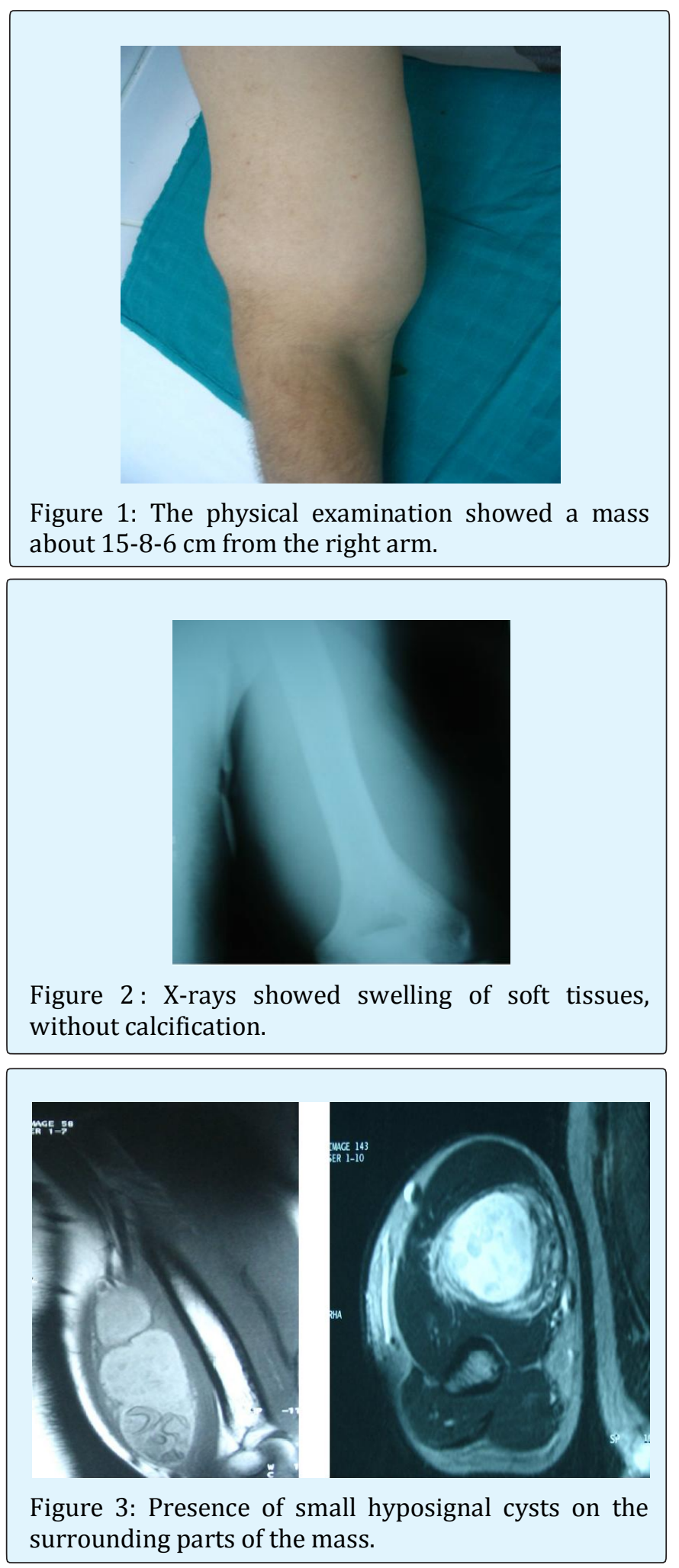

\section{Discussion}

Hydatid disease is an infestation caused by Echinococcus granulosus which rarely ireaches the musculoskeletal system and that is still in temperate zone countries (Mediterranean region and the Middle East, as well as Asia, Australia, New Zealand and North America South) [3-5]. The organs most frequently affected are the liver (75\%), the lungs (15.4\%) (6) that act as natural mechanical filters $[6,7]$. Primary musculoskeletal sites are rare, even in endemic areas (0.5 to $4.7 \%$ ) [8], because of the concentration of lactic acid in the muscle skeletal, and to mechanical factors such as contractile activity that tend to inhibit the embryo from settle in the muscle. Only isolated cases are reported in the literature [5,9]. Primary foci are usually thoracic or abdominal organs from which the diffusion has place either spontaneously or after the operations [10,11]. The best of our knowledge is the second isolated intermuscular hydatid disease, described by Metin Bayram, Akif Sirikci [3]. Most cases of hydatidosis are usually asymptomatic, but may appear several years later, by slow growth of the mass Cystic. Differential diagnosis can be difficult with soft tissue tumors, and Tuberculous masses especially in Morocco which is the first country in the world where tuberculosis ravages the population.

An evaluation by imaging is mandatory in order to avoid any rupture of the cyst during surgery, with consequences of anaphylactic shock and local recurrence especially with iatrogenic procedures, such as puncture percutaneous needle or open biopsy, then the trend of mortality. Recurrence and diffusion is about 70\% [12]. Magnetic resonance imaging is the most useful in the diagnosis and classification of cysts, he has showed a cystic mass, usually containing multiple vesicles (cyst or cysts within a cyst), surrounded by a rim of weak layers intensity and high-signal in T2-weighted images. A high intensity signal on the images weighted in T2 without significant contrast of the plant paramagnetic, suggest a cystic component (Figure 4) [12]. The water lily sign which is a pathognomonic disease sign of hepatic hydatid disease and pulmonary is reported in a single case presentation intermuscularly [12].

Serological tests are not enough to diagnose echinococcosis, sensitivity and specificity are not known, and false negatives occur in 50\% solitary lung cysts [13]. Treatment of muscular echinococcosis requires combination of chemotherapy and surgery [14]; medical treatment is usually mandatory with antihelminthics such as albendazole alone, but the combination with praziquantel was considered superior [7]. Mishandling during surgery can cause a rupture of the cyst with risks 


\section{Journal of Orthopedics \& Bone Disorders}

like anaphylaxis and diffusion to other organs. The principles of surgical excision are similar to a malignant tumor, we proceed to a large excision procedure in the form of a total perkystectomy [14], however, repetitive surgery and accompanying disorders increase postoperative mortality $0.9 \%$ to $3.5 \%$ [15-17].

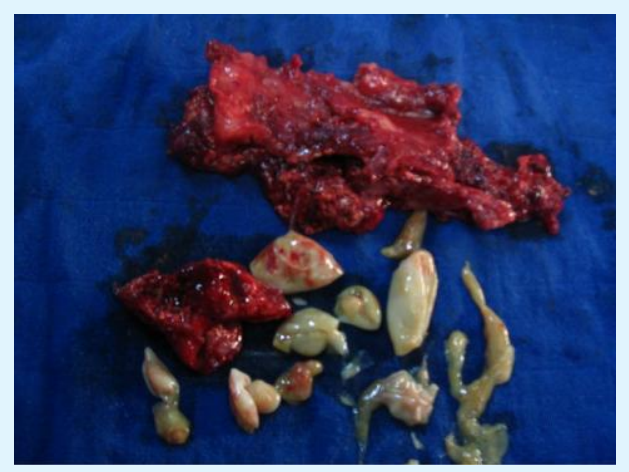

Figure 4: Clinical appearance of the hydatid cyst after resection with the cysts children.

\section{Conclusion}

In summary, muscle infestation with echinococcus is a rare but useful entity to know, more frequently found in rural areas. Intramuscular infestation can be confused with the soft tissue tumors. It can lead to a rupture of the cyst with the risks of anaphylaxis and spread to others organs, that's why it is important to diagnosis it before surgery. Additional pharmacological processing is necessary to achieve a complete cure.

\section{References}

1. Gomori JM, Cohen D, Eyd A, Pomeranz S (1988) Water lily sign in CT of cerebral hydatid disease: a case report. Neuroradiology 30(4): 358.

2. Pasaoglu E, Damgaci L, Tokoglu F, Yildirim N, Ortac Alp A, et al. (1998) CT findings of hydatid cyst with unusual location: infratemporal fossa. Eur Radiol 8: 1570-1572.

3. Bayram M, Sirikci A (2000) Hydatic cyst located intermuscular area of the forearm: MR imaging findings. European Journal of Radiology 36(3): 130132.

4. Guthrie JA, Lawton JO, Chalmers AG (1996) Case report: The MR appearances of primary intramuscular hydatid disease. Clin Radiol 51(5): 377-379.
5. Tatari H, Baran O, Sanlidag T, Gore O, Ak D, et al. (2001) Primary intramuscular hydatidosis of supraspinatus muscle. Arch Orthop Trauma Surg 121(1-2): 93-94.

6. Sanjay M, Subramanian P, Nisha M, Rattan KN, Karwasra RK (2005) Infected primary intramuscular echinococcosis of thigh. Ind J Pediatr 72(9): 799-800.

7. Necati Ormeci A, Ramazan Idilman A, Serdar Akyar B, Murat Palabiyıkoglu A, SahinC, et al. (2006) Hydatid cysts in muscle: a modified percutaneous treatment approach. Int J Infec Dis 11(3): 204-248.

8. Tacal T, Altinok D, Yildiz YT, Altinok G (2000) Coexistence of intramuscular hydatid cyst and tapeworm. AJR Am J Roentgenol 174(2): 575-576.

9. García-Díez AI1, Ros Mendoza LH, Villacampa VM, Cózar M, Fuertes MI (2000) MRI evaluation of soft tissue hydatid disease. Eur Radiol 10(3): 462-466.

10. Combalia A, Sastre-Solsona S (2005) Hydatid cyst of gluteus muscle. Two cases. Review of the literature. J Bone spine 72(5): 430-432.

11. Kazakos CJ, Galanis VG, Verettas DA, Polychronidis A, Simopoulos C (2005) Primary hydatid disease in femoral muscles. J Int Med Res 33(6): 703-736.

12. Comert RB, Aydingoz U, Ucaner A, Arikan M (2003) Water-lily sign on MR imaging of primary intramuscular hydatidosis of sartorius muscle. Skeletal Radiol 32(7): 420-423.

13. Kammerer WS, Schantz PM (1993) Echinococcal disease. Infect Dis Clin North Am 7(3): 605-618.

14. Dudkiewicz I, Salai M, Apter S (1999) Hydatid cyst presenting as a soft-tissue thigh mass in a child. Arch Orthop Trauma Surg 119(7-8): 474-475.

15. Dawson JL, Stamatakis JD, Stringer MD, Williams R (1988) Surgical treatment of hepatic hydatid disease. Br J Surg 75(10): 946-950.

16. Kune GA, Jones T, Sali A (1983) Hydatid disease in Australia. Prevention, clinical presentation, and treatment. Med J Aust 2: 385-388.

17. Koc Z, Agildere AM, Yalcin O, Pourbagher A, Pourbagher M (2004) Primary hydatid cyst in the anterior thigh: sonographic findings. J Clin Ultrasound 32(7): 358-360. 